\title{
Phronesis
}

\section{La situation professionnelle}

Contributions des sciences de l'éducation à l'élaboration d'un objet scientifique

\section{Jean-François Marcel, Frédéric Tupin et Philippe Maubant}

Volume 1, numéro 1, janvier 2012

La situation professionnelle

URI : https://id.erudit.org/iderudit/1006480ar

DOI : https://doi.org/10.7202/1006480ar

Aller au sommaire du numéro

Éditeur(s)

Institut de recherche sur les pratiques éducatives

ISSN

1925-4873 (numérique)

Découvrir la revue

Citer ce document

Marcel, J.-F., Tupin, F. \& Maubant, P. (2012). La situation professionnelle :

contributions des sciences de l'éducation à l'élaboration d'un objet scientifique.

Phronesis, 1(1), 1-4. https://doi.org/10.7202/1006480ar d'utilisation que vous pouvez consulter en ligne.

https://apropos.erudit.org/fr/usagers/politique-dutilisation/ 


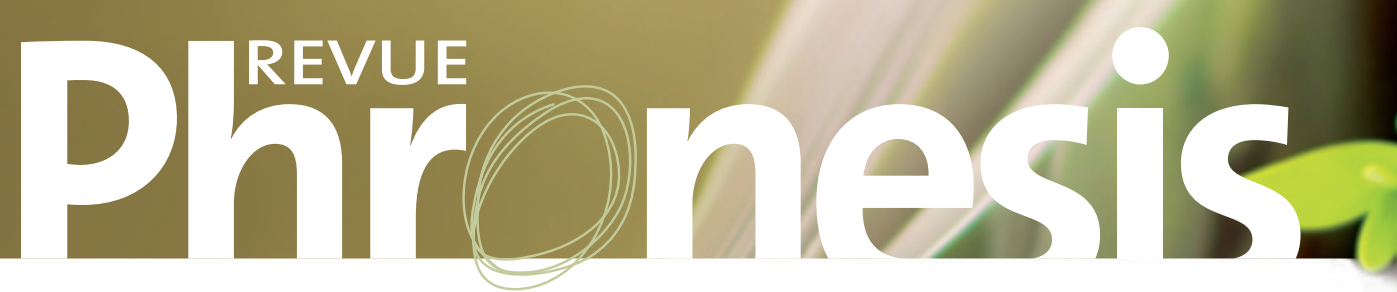

Institut de recherche sur les pratiques éducatives de l'Université de Sherbrooke

\section{LA SITUATION PROFESSIONNELLE \\ Jean-François Marcel* \\ Frédéric Tupin** \\ Philippe Maubant ${ }^{* * *}$}

*ENFA, Université de Toulouse (France), UMR « Éducation, Formation, Travail et Savoirs »

** Université de la Réunion (France), CREN \& LCF UMR8143 CNRS

*** Université de Sherbrooke (Canada), Institut de recherche sur les pratiques éducatives

\section{CONTRIBUTIONS DES SCIENCES DEL'ÉDUCATION}

\section{A L'ÉLABORATION D'UN OBJET SCIENTIFIQUE}




\section{D}

Institut de recherche sur les pratiques éducatives de l’Université de Sherbrooke

\section{Préambule}

Ce premier numéro de la revue Phronesis est issu d'un symposium initié et organisé par les professeurs Tupin de l'Université de Nantes (France), Marcel de l'ENFA de Toulouse (France) et Maubant de I'Université de Sherbrooke (Canada). Ce symposium s'est déroulé du 13 au 16 septembre 2010 dans le cadre du congrès de l'Actualité de la recherche en éducation et en formation à l'Université de Genève. Ce symposium s'inscrit dans les activités scientifiques du Réseau-observatoire international sur la professionnalisation dans les métiers relationnels et de l'interaction humaine initié et soutenu par I'Institut de recherche sur les pratiques éducatives de l'Université de Sherbrooke.

\section{Cadrage théorique}

Nous définirons brièvement et provisoirement la situation professionnelle à l'aide de quelques caractéristiques :

- La situation professionnelle relève conjointement d'une définition (elle n'est pas "donnée", elle est le fruit d'une élaboration par le/ les sujets, qu'il s'agisse d'individu(s), d'un collectif ou d'une institution), et d'une construction (c'est-à-dire d'un ajustement de la situation "projetée" aux contraintes qui pèsent sur sa définition):

- Qu'elle soit vécue, analysée ou observée, la situation est toujours "définie", avec des degrés d'objectivation et d'implicite, certes variables, par un/des sujet(s). La définition de la situation professionnelle est rendue nécessaire par le fait que les significations sociales attribuées à l'objet constitué par l'espace professionnel (représentations, enjeux, valeurs) ne sont pas inhérentes à cet objet indépendamment des acteurs. La situation est donc, sous ce versant, une notion ancrée dans la phénoménologie et marquée par le subjectif.

- Dans le même temps, la situation professionnelle est "construite" et "l'ici et maintenant" de la situation, telle qu'il est pensé par le sujet, est en partie (mais en partie seulement) pré-défini par les éléments structurels dans lesquelles la situation s'insère. La situation est donc en quelque sorte "pré-contrainte" voire "pré-construite".

- La situation professionnelle a une unité (de sens). La construction de la situation par le sujet, dans le rapport dialectique que ce dernier entretient avec les structures dans lesquelles il déploie son action, s'effectue sur la base d'un "découpage" du réel qui n'a rien de contingent (qui a à voir avec sa définition), une sélection des composantes qui font sens à un moment donné et qui permettent de différencier la situation du « reste » d'un environnement qu'elle n'épuise pas. Ainsi, telle la tragédie classique, la situation se caractérise par une unité relative de temps, de lieu et d'acteur(s) (et nous pourrions rajouter d'action). La relativité de cette unité tient à la question du sens (propre au sujet) et aux démarches (en partie implicites) de définition et de construction de cette situation. Pour autant, la définition et la construction de la situation professionnelle ne s'opèrent pas nécessairement sur un mode «actif», elles ne sont pas nécessairement portées par des stratégies délibérées mais peuvent être assumées par le sens pratique issu d'un "système social réflexe" porteur d'attitudes incorporées. Dans ce cas, la définition et la construction de la situation relèvent d'un processus implicite, tacite ou infra-conscient.

- La situation professionnelle est action. La situation est action d'abord parce qu'elle est définition et qu'elle est construction, mais aussi parce qu'elle est liée à l'agir (au travers du projet qui en porte ses finalités). Il n'y a pas de situation sans action comme il n'y a pas d'action sans situation. La finalité de l'action est indissociable du sens de la situation pour le sujet : en agissant le sujet transforme la situation, il la redéfinit par une dynamique d'ajustement du sens à la finalité de l'action et la reconstruit en ajustant la finalité à la fois à l'avancée de l'action et à l'évolution du contexte mais aussi au sens initial de la situation désirée "projetée". De plus, dans le cas d'une situation professionnelle, cette action est caractérisée par le cadre professionnel (structure, finalités, valeurs, culture, lois, règles, normes, prescriptions, hiérarchie, etc.). 
En résumé, la notion de situation professionnelle que nous proposons de mettre en débat dans ce numéro de la revue Phronesis apparaît comme la résultante :

- D'une définition, ancrée dans une situation « projetée » par le sujet et porteuse de sens,

- D'une construction, dans un système conjoint de contraintes et de ressources (internes et externes) actualisé par les choix et la dynamique interactive des acteurs concernés.

In fine, nous pourrions concevoir la situation comme la production d'un sujet "centre du monde" immergé dans un champ de rapports dialectiques entre, "l'ici et maintenant" (habité par la micro-société constituée par le sujet) et "les différents cercles contextuels" (établissement, entreprise, réseaux locaux ou régionaux, institution, etc.) qui marquent de leur empreinte les conditions d'expression du sens pratique et qui relèvent d'une hybridation entre habitus individuel, habitus de classe et habitus professionnel.

Simultanément et conjointement aux contraintes (relatives) qu'imposent les structures externes, à l'empreinte des contextes, au filtre des habitus, le sujet est appelé, par la mise en œuvre de sa rationalité (fréquemment limitée), à interpréter ce qui se produit dans le «micro-contexte situationnel» socio-professionnel dans lequel il évolue. Cette construction (qui ne s'émancipe jamais tout à fait de la définition initiale de la situation) ne peut s'élaborer que via une dynamique interactive avec les alter concernés, sur le mode d'une recherche croisée d'anticipations des motifs de «l'autre» et d'une adaptation à ces intentions supposées. Elle ne peut se réaliser, sur le mode de la négociation, que dans un dosage subtil entre actions/évaluations indépendantes et actions/évaluations inter-dépendantes.

\section{Objectifs scientifiques}

Ce numéro 1 de la revue Phronesis a choisi de mettre en débat la notion de «situation professionnelle » en provoquant le dialogue entre des recherches en Sciences de l'éducation qui l'investissent. Leur diversité est manifeste, qu'il s'agisse des cadres théoriques convoqués, des méthodologies mobilisées, des champs professionnels investigués voire des visées poursuivies et des postures adoptées.

L'hypothèse qui sous-tend ce numéro consacré au construit de situation professionnelle est donc que, au delà de ces diversités, des ces fondements épistémologiques, disciplinaires, théoriques variés, le dialogue scientifique est possible. Il valorise une ambition de mettre en évidence une perspective multiréférentielle, interdisciplinaire et interprofessionnelle de la professionnalisation dans les métiers adressés à autrui. C'est pourquoi, outre les textes des auteurs, nous avons souhaité aussi mobiliser un grand témoin, le professeur René Amigues, de l'Institut universitaire de formation des maîtres d'Aix-Marseille. Notre collègue a pensé le déroulement du symposium comme une "situation professionnelle » réunissant des enseignants-chercheurs et qui a accepté d'en rendre compte dans le cadre d'un texte qui clos ce numéro. L'objectif de ce numéro est de dégager de cette diversité et de cette confrontation quelques éléments permettant de théoriser plus avant la notion de situation professionnelle » et de contribuer à la construire en tant qu'objet scientifique.

Pour soutenir cette ambition, le texte de Driss Allaoui s'intéresse à la situation d'accompagnement socio-professionnel. Sous l'angle de l'ethnologie, l'auteur analyse les dispositifs d'accompagnement de populations en difficulté d'insertion sur l'île de la Réunion. II analyse tout particulièrement les pratiques d'accompagnement de professionnels du social. Il révèle combien les représentations et les postures de l'accompagnement qui en découlent, tendent à déterminer la situation et les pratiques professionnelles mises en place. Le texte de Catherine Guillaumin, quant à lui, traite de la situation professionnelle à partir d'une analyse d'une formation par alternance de professionnels en soins infirmiers. En étudiant les récits des situations professionnelles rencontrés et vécues par les étudiants en formation, Catherine Guillaumin propose l'esquisse d'un modèle en construction de la situation professionnelle comme moment critique dans l'action du sujet en interaction avec autrui. Jean-François Marcel présente un cadre de recherche : le travail partagé des enseignants. Ce texte soumet à l'empirie l'hypothèse d'une co-élaboration de la situation professionnelle. L'auteur analyse le processus de reconstruction d'une séance d'enseignement (situation de référence) au cours d'une séance de conseil pédagogique (situation support) caractérisée par l'interaction entre une enseignante débutante et un formateur. Paul Olry mobilise la psychologie ergonomique appliquée à la formation professionnelle des adultes. Il s'intéresse à la situation professionnelle, dans la mesure où, selon lui, elle ne se superpose pas nécessairement à la situation de travail. Si cette dernière est travaillée par de nombreuses disciplines, la situation professionnelle intéresse avant tout la pratique. L'auteur interroge donc la part d'invariance d'une situation professionnelle 
dans la tension entre sa fonction sociale et l'usage opératif nécessaire pour la comprendre. Prenant appui sur le cas des conseillers agricoles, conduits à réorienter leurs pratiques sur les raisonnements liés aux évolutions agroenvironnementaux et sur la capacité des agriculteurs à les construire, les données exploratoires présentées dans ce texte montrent les glissements de statut donné à la situation. Jean-Luc Rinaudo cherche à cerner comment les technologies de l'information et de la communication (TIC) utilisées dans les pratiques des enseignants contribuent à redessiner les situations professionnelles d'enseignement. L'auteur considère que ce projet de définition de la situation professionnelle d'enseignement peut aider à mieux lire et comprendre les pratiques enseignantes elles-mêmes. Philippe Maubant, Lucie Roger et Bernard Mercier inscrivent la visée d'apprentissage comme constitutive des situations professionnelles. Selon ces auteurs, la situation d'apprentissage professionnel pourrait être, sous certaines conditions, la figure didactico-pédagogique d'un dispositif de formation ayant pour ambition la professionnalisation des futurs enseignants. En convoquant Bachelard pour définir l'acte d'apprendre, les auteurs revendiquent la nécessité d'inscrire le processus de construction des connaissances au cœur des situations professionnelles. Patrick Mayen, inscrit sa réflexion dans l'héritage de la didactique professionnelle, discipline qui se propose d'apprendre des situations. En faisant dialoguer l'analyse du travail, l'analyse de l'activité et les différentes situations, l'auteur définit la situation d'apprentissage professionnel. Il utilise cette perspective pour penser autrement la formation, conçues dès lors, structurée et organisée autour des situations. Frédéric Tupin et Laetitia Sauvage, en s'intéressant aux situations d'enseignement-apprentissage, interrogent les contextes et conditions d'exercice dela profession enseignante. En recourant aux travaux de Giddens et de Brofenbrenner, Frédéric Tupin et laetitia Sauvage explore la notion de situation professionnelle en la confrontant à une autre notion, celle de contexte. En cherchant à contextualiser l'analyse de la pratique enseignante, ils cherchent à définir l'activité enseignante non pas comme une action en situation mais comme une action contextualisée en situation.

Ce premier numéro de la revue Phronesis ne prétend pas arrêter, de manière définitive, le cadre de lecture et d'interprétation de la notion de situation professionnelle. Mais les différents textes permettront de toute évidence aux lecteurs de mieux saisir les délicates nuances entre les différentes acceptions scientifiques du concept de situation professionnelle et ses différents sens et usages au regard des enjeux de recherche et des enjeux de pratique. 